\title{
El Sistema de Posicionamiento Global - GPS ${ }^{1}$
}

Jorge R. Rey ${ }^{2}$

El Sistema de Posicionamiento Global ("Global Positioning System" - GPS) es un sistema de navegación compuesto de una flotilla de satélites puestos en órbita por el Departamento de Defensa de los Estados Unidos, y sus estaciones en tierra firme. Funciona continuamente en todas partes del mundo y es disponible a todos libre de cargos. Con orígenes en aplicaciones militares secretas, GPS se ha convertido en parte de nuestra vida cotidiana.

\section{Historia}

El GPS surgió debido a la necesidad de las fuerzas armadas de tener un sistema de navegación preciso y que funcionara en aplicaciones diversas. El desarollo de la tecnología de GPS descanza en progresos en ciencias físicas, en la electrónica, en ciencias de materiales y en muchas otras, pero fué el desarollo de dispositivos extremadamente precisos para medir el tiempo - relojes atómicos, junto con progreso en la tecnología espacial, que en realidad hicieron posible el GPS.

Medida de Tiempo con Precisión - Todos los átomos emiten ondas electromagnéticas cuando cambian su estado energético debido a la reorganización de sus electrones. Estas ondas se conocen como frecuencias resonantes y son extremedamente precisas y características de cada tipo de átomo. En 1944, I. I. Rabi, ganador del Premio Nobel de ese año por el desarollo de la técnica de resonancia magnética para medir las frecuencias resonantes de los átomos, sugirió que debido a la precisión de las resonancias atómicas, ellas podrían ser usadas para crear relojes extraordinariamente precisos.

En 1948, la Oficina Nacional de Normas en los Estados Unidos, fabricó el primer reloj atómico. Este utilizaba moléculas de amoniaco en vez de átomos de cesio, y el tubo de haz era un tubo de cobre envuelto sobre la esfera del reloj. Nunca fué usado ya que era menos fiable que un común reloj de cuarzo, con error de un segundo cada cuatro meses. El primer reloj atómico práctico fué construído en 1955 y ocupaba una sala completa del Laboratorio Nacional de Física, en Gran Bretaña. Tenía una precisión de un segundo en 300 años. En 1956 se fabricó una versión portable, el "Atomichron", que podía moverse facilmente de sitio a sitio.

1. Este documento, ENY-728S, es uno de una serie de publicaciones del Departamento de Entomología and Nematología Servicio de Extensión Cooperativa de la Florida, Instituto de Alimentos y Ciencias Agrícolas, Universidad de la Florida. (UF/IUFAS). Fecha de primera publicación: April 10, 2006. Visite nuestro sitio web EDIS en <http://edis.ifas.ufl.edu>.

2. Jorge R. Rey, professor, Entomology and Nematology Department, Florida Medical Entomology Laboratory, Vero Beach, Cooperative Extension Service, Institute of Food and Agricultural Sciences. University of Florida, Gainesville, FL 32611

El uso de nombres comerciales citados en esta publicación es sólo con el propósito de brindar información específica. El Instituto (UF/IFAS) no garantiza los productos nombrados, y las referencias a ellos en esta publicación no significa nuestra aprobación a la exclusión de otros productos de composición comparable.

The Institute of Food and Agricultural Sciences (IFAS) is an Equal Opportunity Institution authorized to provide research, educational information and other services only to individuals and institutions that function with non-discrimination with respect to race, creed, color, religion, age, disability, sex, sexual orientation, marital status, national origin, political opinions or affiliations. U.S. Department of Agriculture, Cooperative Extension Service, University of Florida, IFAS, Florida A. \& M. University Cooperative Extension Program, and Boards of County Commissioners Cooperating. Larry Arrington, Dean 
Otras versiones del reloj atómico fueron creadas en poco tiempo. Estos nuevos dispositivos empleaban frecuencias resonantes de cesio, rubidio, y el máser de hidrógeno, el cual utilizaba átomos de hidrógeno y moléculas de amoniaco. Los relojes basados en rubidio son más pequeños y económicos que los de cesio, pero son menos precisos.

En 1967, el segundo fue redefinido oficialmente como la duración de 9,192,631,770 ciclos del cesio-133. La norma de tiempo en los Estados Unidos es el reloj atómico NIST-7, en el Instituto Nacional de Normas y Tecnología, en Boulder, Colorado, cuya precisión se estima ser un segundo en tres millones de años! Progresos tecnológicos continuan permitiendo el desarollo de relojes atómicos más y mas pequeños y económicos (Cuadro 1).

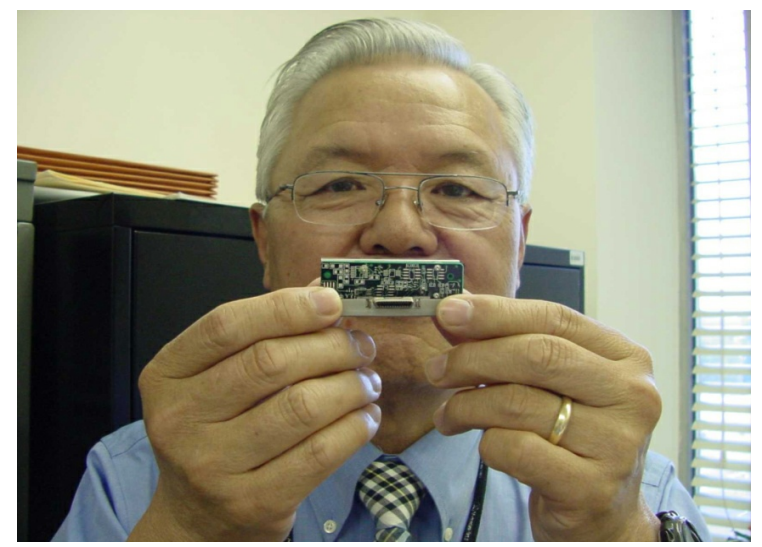

Cuadro 1. Dr.John Kimcon con un reloj atómico de rubidio ultra miniaturizado. Credits: Office of Naval Research

La Era Espacial - La semilla de la navegación por satélites fué sembrada casi al mismo tiempo que el comienzo de la era espacial. Cuando los soviéticos lanzaron el primer satélite en orbita terrestre, el Sputnik I, en 1957, investigadores en el Laboratorio de Física Aplicada de Johns Hopkins en Baltimore, descubrieron que debido a que ellos sabían exactamente su posición en la tierra, ellos podían calcular la posición exacta del satélite midiendo el corrimiento en la señal de radio transmitida por Sputnik según se acercaba y alejaba de ellos. El corrimiento en la frecuencia, conocido como el efecto Doppler o el corrimiento Doppler, se conocía desde el año 1842, cuando Christian Doppler propuso que la frecuencia de ondas en movimiento con respecto a un observador, aparentemente aumenta según la fuente de las ondas se acerca al observador, y disminuye según se aleja. Solo tomó un pequeño salto de intuición averiguar que lo opuesto también era verdad; que uno puede determinar su posición exacta en la tierra si se conoce la posición exacta del satélite.

En 1965, la Marina Estadounidense desarolló el sistema "Transit" debido a la necesidad de tener un sistema de navegación fiable para submarinos que podían mantenerse sumergidos por semanas y meses. El sistema estaba compuesto de seis satélites en órbitas polares. La posición era determinada usando el corrimiento Doppler. Usando este sistema, se puede determinar la posición en dos dimensiones en 6 a 10 minutos con una precisión de 25 metros.

Durante la década de los 60, varias ramas de las fuerzas armadas trabajaron en sus propias versiones de sistemas de navegación pos satélite. En el 1973, el Departamento de Defensa, ordenó a las diferentes ramas que cooperaran en el desarollo de un sistema unificado. El resultado fué el Sistema de Posicionamiento Global "Navstar", el cual depende de satélites que llevaban relojes atómicos a bordo (un concepto que fué probado con anterioridad en un programa de la Marina llamado "Timation"), estaciones terrestres que controlan el sistema, y posicionadores (receptores) para el usuario que no dependen de relojes atómicos.

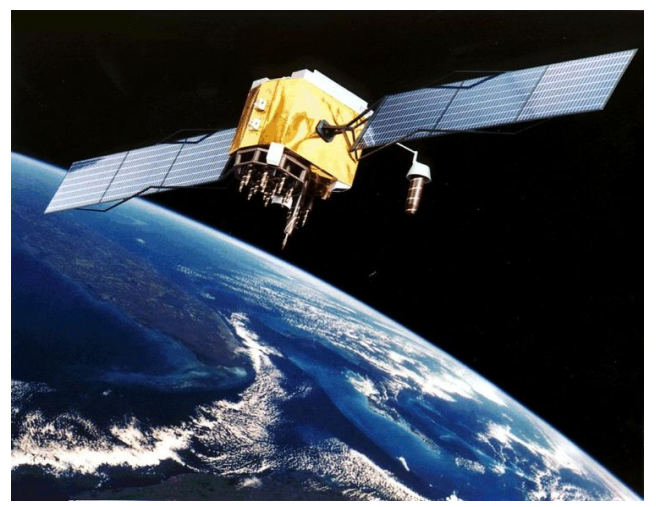

Cuadro 2. Concepto artístico de un satélite NAVSTAT. Credits: NASA

El primer satélite de GPS fue lanzado en el 1978. Comenzando en el 1989, una segunda generación de satélites (Satélites de Bloque II) fué puesta en servicio. El sistema alcanzó operación plena en el año 1995. En el presente, la flotilla de satélites de 
GPS consiste en, por lo menos, 24 satélites Bloque II (Cuadro 2). En 1983, luego de que una aeronave Coreana de pasajeros fué derribada por los soviéticos en su espacio aéreo, el presidente Ronald Reagan declaró que el sistema GPS sería disponible para usos civiles luego de que se completara.

Debido a que el sistema GPS fué desarollado principalmente para aplicaciones militares, errores de cronometraje (disponibilidad selectiva, "selective availability" - SA) fueron aplicados a las señales de GPS, lo cual limitaba la precisión de posicionadores no militares. Durante la guerra del Golfo Persa en 1991, el sistema GPS se había echo tan indispensable, que no habíán suficientes posicionaderes (receptores) militares para las tropas, por lo cual el Departamento de Defensa tuvo que usar posicionadores civiles y eliminar temporariamente la SA. La SA global fué eliminada permanentemente en el año 2000, pero el servicio militar Estadounidense aún puede introducir errores en las señales en extensiones geográficas limitadas.

\section{¿Como Funciona el GPS?}

GPS depende en que cada satélite en la constelación transmita su posición exacta y una señal de tiempo extremadamente precisa a los recibidores en la tierra. Dada esta información, los receptores GPS pueden calcular su distancia al satélite, y combinando esta información de cuatro satelites, el recibidor puede calcular su posición exacta usando un proceso llamado trilateración.

Trilateración - Si uno conoce la distancia a un satélite, uno sabe que su posición se encuentra dentro de una esfera con centro en el satélite y con un radio igual a la distancia (Cuadro 3A).

Si uno obtiene la misma información de un segundo satélite, puede estrechar su posible posición al área en tres dimensiones que tienen en común las dos esferas (región matizada, Cuadro 3B). Si se añade información de un tercer satélite, se puede precisar aún más la posición a los dos puntos donde las tres esferas cruzan (cuadrados pequeños, Cuadro 3C). Para determinar cual de los dos puntos representa nuestra posición actual, podemos tomar una cuarta medida, pero

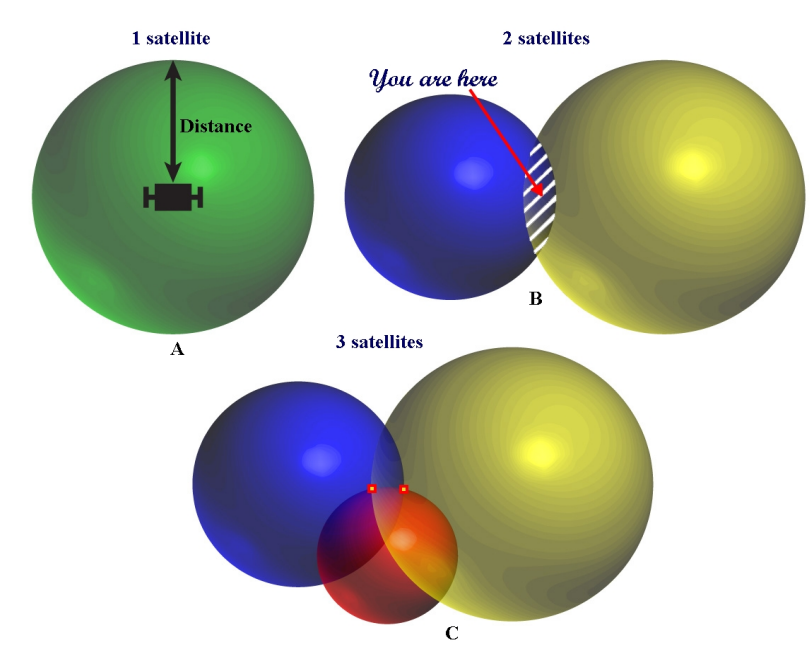

Cuadro 3. Calculando la posición usando tres satélites. Credits: Jim Newman

generalmente uno de los dos puntos obtenidos de tres satélites representa una posición absurda (por ejemplo en el espacio abierto) o con movimiento imposiblemente rápido, por lo cual se puede eliminar sin necesidad de la cuarta medida. Sin embargo, la cuarta medida aún es necesaria, lo cual se explica seguido.

El cuarto satélite - La distancia a los satélites se calcula midiendo el tiempo que toma a la señal a llegar del satélite al recibidor (Distancia = velocidad $\mathrm{X}$ tiempo). Debido a que las señales de radio viajan a la velocidad de la luz (186,000 millas por segundo) los tiempos en tránsito de satélites a recibidores son extremadamente pequeños y se necesitan dispositivos de cronometraje extremadamente precisos para medirlos con exactitud, por lo cual surge la necesidad de llevar relojes atómicos en los satélites. Sin embargo, los receptores no llevan relojes atómicos lo cual introduce errores en ese lado del sistema y aún errores de cronometraje pequeños pueden resultar en grandes errores de posición. Aquí es donde entra en juego la cuarta medida.

Si las cuatro medidas son exactas, la esfera definida por la cuarta medida debe cruzar las otras tres en un punto que representa la posición actual. Si existen errores, la cuarta esfera no cruzará a todas las otras. Debido a que el error del recibidor es el mismo para las cuatro medidas, un ordenador en el recibidor puede calcular una corrección que haga que las cuatro esferas cruzen, y aplicar la corrección a las medidas para obtener la posición correcta. 
Otros errores - Otros errores tienen influencia en las medidas de las señales de los satélites GPS, entre ellos están la interferencia atmosférica y reflejos de obstáculos en la tierra como árboles y edificios. Se utilizan varios métodos como la corrección por frecuencia doble, filtros de señales, y modelos matemáticos para disminuir sus efectos.

Aumento en la precisión - Nuevos métodos están en desarrollo contínuo para hacer los sistemas GPS más precisos y confiables. En el presente, los más comunes son el GPS diferencial (DGPS), y el Sistema de Aumento en Areas Amplias ("Wide Area Augmentation System" - WAAS). El DGPS basicamente utiliza estaciones basadas en tierra, cuya ubicación se conoce precisamente, para recibir las señales de los satélites, aplicar correcciones (como se conoce su ubicación exacta, las estaciones pueden determinar los errores en las señales), y diseminarlas de modo que receptores adecuadamente equipados las puedan recibir y usarlas para corregir sus propias señales. WAAS es un sistema de satélites segundario desarrollado por la Administración Federal de Aviación que no solo transmite señales de GPS, sino también monitorea y reporta el estado de los satelites de GPS, y transmite información de DGPS. El sistema solo es disponible sobre Norteamérica y el Océano Pacífico.

\section{Usos para el GPS}

El desarrollo de posicionadores de GPS precisos y a precios razonables (Cuadro 4), y la miniaturización de componentes electrónicos, han hecho que el GPS se encuentre disponible a casi todo el mundo, lo cual ha facilitado que el GPS esté convirtiendose en una necesidad en muchas facetas de la vida cotidiana.

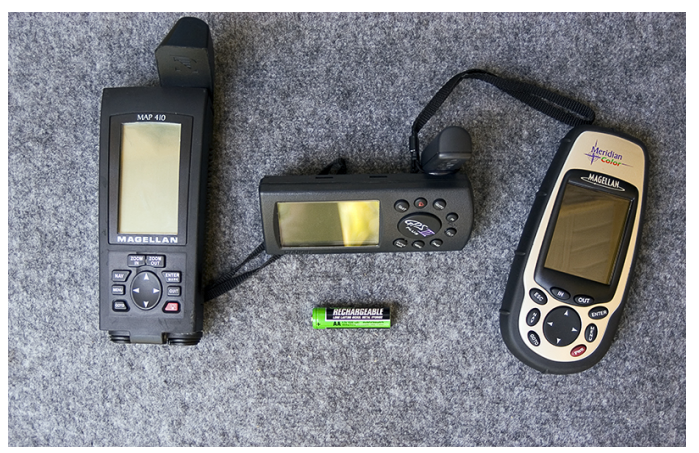

Cuadro 4. Posicionadores de GPS precisos están disponibles hoy en día a precios razonables.
Enumerar todas las aplicaciones para el GPS sería una labor imposible. Seguido se dan solo algunos ejemplos de aplicaciones de GPS en el mundo moderno.

Agricultura - La navegación por satélite puede ayudar a los agricultores a aumentar su producción y a mejorar la eficiencia de sus métodos de cultivo. Sistemas de GPS ubicados en los tractores y en otros vehículos agrícolas, junto con sofisticados sistemas de información geográficos utilizan varios métodos para recoger datos sobre las condiciones del suelo, humedad, temperatura y muchas otras variables. Con esta información, que el sistema puede controlar muchos apectos de la operación agrícola, por ejemplo, la intensidad de siembra, la aplicación de fertilizantes e insecticidas, los patrones de riego y mucho más. Estos sistemas también puede ayudar a los agricultores a mantenerse al tanto de la operación de sus fincas, manteniendo registros de rendimiento, historia de aplicación de productos químicos, analyses del suelo, registros de pérdidas y ganancias por terreno, y muchos otros. Para obtener más información sobre los usos de GPS en agricultura consulte el vínculo siguiente: http://deathstar.rutgers.edu/projects/gps/web_page/ web_page.html

Navegación en Tierra y Mar - Además de sus usos directos para determinar posición usando sistemas de navegación a bordo, GPS se utiliza para mejorar la precisión de cartas marinas, para guiar sistemas de auto-piloto, para marcar objetos sumergidos como obstrucciones o su sito favorito de pesca, y para determinar la posición exacta de naves en alta mar. Agencias de socorro y primeros auxílios dependen de sistemas de GPS para reducir el tiempo en tránsito a emergencias. Empresas de fletes y mudadas usan sistemas de GPS para mantenerse al tanto de la ubicación de sus vehículos, para planificar más eficientemente sus horarios de recogidas y entregas, y para determinar las tablas de mantenimento para los vehículos. El GPS también se está usando para mantenerse al tanto de la posición de vehículos y otras propiedades móbiles, y en sistemas para ayuda directa al motorista como el OnStar. 
Usos Militares - Además de las aplicaciones más comunes tal como la navegación general, los servicios militares utilizan el GPS en gran variedad de aplicaciones incluyendo dirigir proyectiles y "bombas inteligentes" a sus blancos, para organizar el despliegue de tropas, para la coordinación en el campo y muchas otras.

Mapas y Agrimensura - El GPS permite la construcción de mapas y cartas más precisas, y es usado rutinariamente por agrimensores para planear proyectos y localizar marcadores cadastrales, límites, estructuras, y rasgos naturales.

Ciencias - Las aplicaciones de GPS en las ciencias son inumerables. El GPS es especialmente valioso para investigadores de campo, para construir mapas y localizar sus estacioneds de muestreo, para definir límites de habitáculos, para analisis espacial de rasgos naturales, para seguir a poblaciones de animales y muchas otras. GPS tambien es usado ampliamente en la seismología, física, ciencias del espacio y en muchas otras ramas de la ciencia.

Recreación - GPS es usado por operadores de embarcaciones para mantener el curso y para regresar a sitios favoritos, por ciclistas y naturistas para mantenerse al tanto de sus posiciones y rutas, y por grupos de viaje para compartir información sobre viajes y rutas. Un nuevo "deporte" es el "geocaching" lo cual es, basicamente una búqueda de tesoros guiada por GPS. Los jugadores utilizan el GPS para viajar a coordinadas geográficas específicas y encontrar objetos escondidos por otros jugadores.

\section{Referencia de Tiempo - GPS se usa} frecuentemente como un cronómetro fiable. Por ejemplo, el GPS puede ser usado para sincronizar múltiples instrumentos científicos desplegados en el campo, o para cualquier experimento que requiera sincronización precisa. La tecnología de GPS también se esta usando para sincronizar torres de comunicaciones celulares, redes de telecomunicación y muchas otras. Cronometraje por GPS se usó en las olimpiadas por primera vez en los juegos del 2000.

\section{Preparación del Posicionador (receptor)}

En la mayoría de situaciones, GPS será utilizado para determinar posición exacta, para seguir direcciones a un punto deseado, para determinar la distancia y dirección a un destino deseado, para medir altitud, para marcar automaticamente una ruta de viaje de modo de que se pueda retrazar, para indicar la dirección deseada de viaje, o puede usarse como una brújula común.

Además de proveer información geográfica, muchos posicionadores modernos pueden enseñar graficamenet una superposción del terreno e incluir su posición, rutas y puntos de destino (Cuadro 5). Sin embargo, a menudo hay que comprar los datos del terreno de su área por separado.

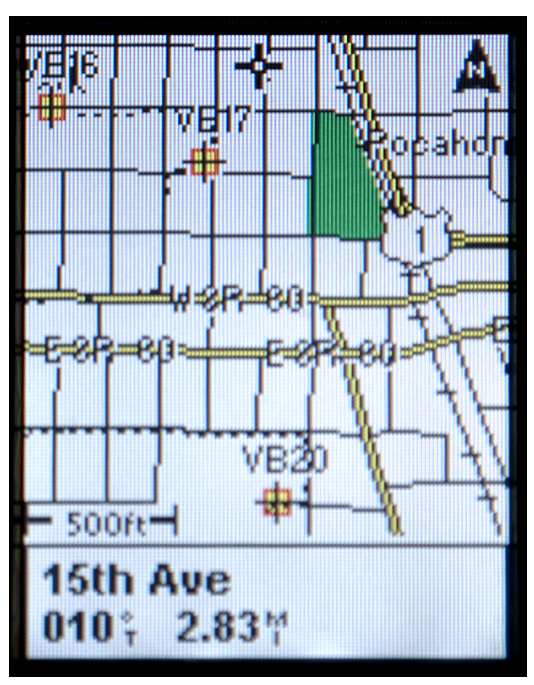

Cuadro 5. Superposiciones del terreno están disponible para muchos posicionadores GPS.

La operación de los posicionadores GPS varía dependiendo del tipo de posicionador, el fabricante, y la labor que se desee completar. Los manuales del usuario de cada unidad contienen instrucciones detalladas para su uso las cuales se deben estudiar cuidadosamente. La mayoría de los fabricantes de unidades GPS también mantinenen páginas web con instrucciones detalladas y sugerencias para el uso de sus unidades. Seguido se explican algunos de los pasos iniciales necesarios para la operación de un posicionador GPS.

Información personal - La preparación de su posicionador comienza con el registro de su 
información personal, tal como su nombre y localización, y de información y controles generales tales como la fecha, la fuente de tipos, la claridad de la pantalla, etc.

Pantallas informativas - Los posicionadores GPS modernos pueden exponer gran cantidad de información, y la mayoría ofrecen opciones sobre el tipo de información incluída en cada pantalla (Cuadro 6). Aprenda como desplegar las diferentes pantallas y como cambiar la información mostrada en ellas para siempre tener a mano la información deseada.

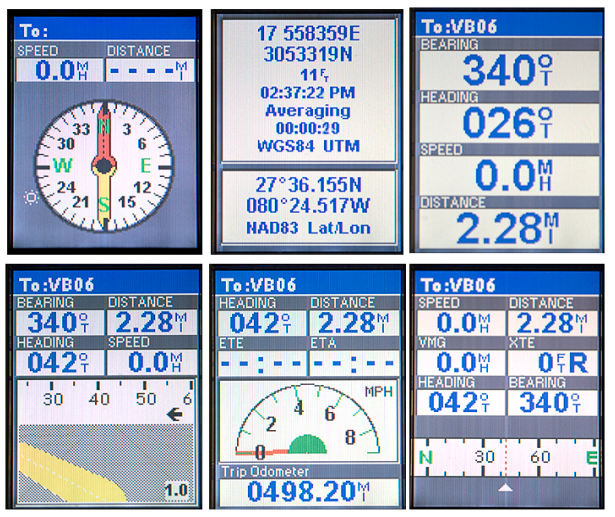

Cuadro 6. Aprenda como exhibir las diferentes pantallas informativas y como cambiar la información en ellas.

Seleccione un datum cartográfico - Un datum cartográfico es una superficie de referencia definida matematicamente la cual aproxima la forma de la Tierra en sitios específicos. El datum se usa para calcular las coordenadas en cualquier mapa, carta de navegación, o sistema de agrimensura. En otras palabras, un datum cartográfico es una fórmula matemática que transfiere las coordenadas en un mapa plano a la forma actual de la Tierra y vice versa. En Norteamérica los datums más comunes son los NAD27, NAD83, y WGS83. El WGS83 se está convirtiendo rapidamente en la norma mundial, y en Norteamérica es practicamente idéntico al NAD83 para casi todas las aplicaciones normales. El NAD27 ha sido reemplazado por el más moderno y preciso NAD83, pero aún existen muchos mapas viejos basados en el datum NAD27

Si usted trabaja con un mapa impreso, asegúrese de que su posicionador GPS esté fijado al mismo datum que el mapa (el cual tendrá esa información impresa en algún sitio). También, si usted está siguiendo puntos en una ruta, o se encuentra navegando hacia unas coordenadas predeterminadas, asegúrese que su posicionador está usando el mismo datum.

\section{Seleccione un sistema de coordenadas - $\mathrm{Su}$} unidad GPS puede reportar información posicional en dos fomatos principales, lat-lon y UTM. Lat-lon es el ya familiar sistema de latitud y longitud. en el cual las coordenadas son reportadas en grados, minutos y segundos norte o sur (latitud) y este $\mathrm{u}$ oeste (longitud), por ejemplo: $33^{\circ} 12^{\prime} 29^{\prime \prime} \mathrm{N}$, $80^{\circ} 23^{\prime} 59^{\prime \prime} \mathrm{W}$ (o en minutos decimales, $\left.33^{\circ} 12.4833^{\prime} \mathrm{N}, 80^{\circ} 23.9833^{\prime} \mathrm{W}\right)$. En el sistema UTM ("Universal Transverse Mercator" - Mercator Transversal Universal), el mundo está dividido en 60 zonas de igual tamaño (también llamadas "husos") de oeste a este, comenzando en el meridiano $180^{\circ}$. La posición se describe en kilómetros al este del principio de cada zona ("easting") y lejos del ecuador ("northing"). En el hemisferio Norte, el northing es la distancia al ecuador mientras que en el hemisferio sur es la distancia al Polo Sur. Para poder interpretar la posición globalmente, hay que saber en que hemisferio y zona está localizado el punto de interés.

Las coordenadas UTM aparecen como una serie de números comenzando con el número de la zona y luego el easting y el northing en ese orden (Cuadro 7).

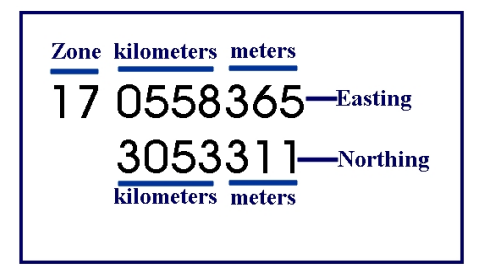

Cuadro 7. Presentación típica de coordenadas UTM (sin las notas textuales).

En el ejemplo ilustrado en el cuadro 7, la posición indicada está 558 kilómetros y 365 metros al este del principio de la zona 17 y 3,053 kilómetros y 311 metros al norte del ecuador. La mayoría de los mapas modernos incluyen en superposición una cuadrilla de UTM a escala de kilómetro, lo cual hace más fácil la localización de puntos con coordenadas UTM. 
Adquisición de satelites - El primer paso en el uso actual de su receptor GPS es encender el posicionador y dejarlo que encuentre (adquiera) por lo menos cuatro satélites. Luego que esto ocurra, su posicionador "conoce" su posición exacta y está listo para funcionar, Mientras la unidad adquiere los satélites, la pantalla casi siempre enseña una "carta del cielo" circular donde se indican los satélites que están disponibles en el momento, y un gráfico indicando cuales satélites se están recibiendo y la calidad de la señal (Cuadro 8). Esta pantalla se puede consultar en cualquier momento para verificar la calidad de recepción.

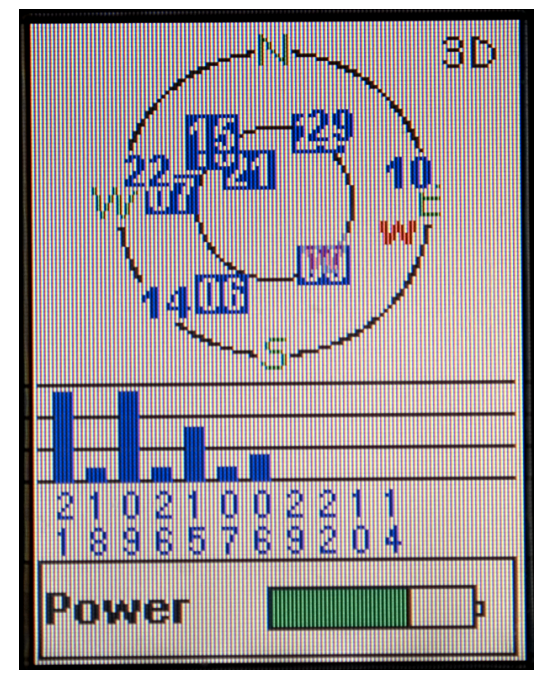

Cuadro 8. Pantalla que enseña los satélites disponible y la calidad de recepción.

La primera vez que un posicionador es usado, o si se lleva a localidades geográficas muy diferentes a la anterior, el proceso de adquirir los satélites se puede hacer más eficiente si se ejecuta una calibración inicial, lo que basicamente consiste en indicarle al posicionador a grandes rasgos, el área general donde se encuentra.

Tenga un plan de reserva - Nunca cuente solamente con su posicionador GPS para navegar en áreas desconocidas. Como todo aparato mecánico/electrónico, las unidades GPS pueden fallar (una "falla" muy común es agotar las baterías, así que siempre lleve baterías de repuesto cuando use su posicionador). En muchos sitios, tal como bajo cubierta frondosa de árboles, la recepción no será buena, y por lo tanto tendrá que utilizar otros métodos de navegación, tal como mapa y brújula, hasta que pueda readquirir la recepción.

\section{Additional Web Resources}

Geospatial Resource Portal - Global

Positioning System -

http://www.gisdevelopment.net/tutorials/ tuman004.htm.

Grant F. Walton Center for Remote Sensing and Spatial Analysis -

http://deathstar.rutgers.edu/projects/gps/web_page/

web_page.html.

Introduction to GPS Applications -

http://www.redsword.com/gps/old/index.htm.

Lowrance, What is GPS? -

http://www.lowrance.com/Tutorials/GPS/

gps_tutorial_01.asp.

Map Tools -

http://www.maptools.com/FreeTools/UTMtools.html.

OnStar Technology -

http://www.onstar.com/us_english/jsp/explore/

onstar_basics/technology.jsp. 